\title{
Germanica
}

\section{À la recherche de l'identité européenne : le « rapport » du Finlandais Jörn Donner sur l'Europe centrale en 1962}

Auf der Suche nach der europäischen Identität : Jörn Donners «Bericht über die Donauländer»

\section{Annie Bourguignon}

\section{(2) OpenEdition}

\section{Journals}

Édition électronique

URL : http://journals.openedition.org/germanica/2192

DOI : 10.4000/germanica.2192

ISSN : 2107-0784

Éditeur

Université de Lille

\section{Édition imprimée}

Date de publication : 31 décembre 2001

Pagination : 77-89

ISBN : 9782913857063

ISSN : 0984-2632

\section{Référence électronique}

Annie Bourguignon, « À la recherche de l'identité européenne : le « rapport » du Finlandais Jörn Donner sur l'Europe centrale en 1962 », Germanica [En ligne], 29 | 2001, mis en ligne le 20 septembre 2013, consulté le 06 octobre 2020. URL : http://journals.openedition.org/germanica/2192 ; DOI : https:// doi.org/10.4000/germanica.2192

Ce document a été généré automatiquement le 6 octobre 2020.

(c) Tous droits réservés 


\title{
À la recherche de l'identité européenne : le « rapport » du Finlandais Jörn Donner sur l'Europe centrale en 1962
}

\author{
Auf der Suche nach der europäischen Identität : Jörn Donners « Bericht über die \\ Donauländer »
}

Annie Bourguignon

1 Parmi les « écrivains voyageurs », les " écrivains-reporters » constituent une catégorie particulière. Le reportage obéit à des règles nettement plus contraignantes que celles auxquelles est soumis le récit de voyage en général, car il doit donner au lecteur une image d'un lieu déterminé à un moment déterminé, en privilégiant les phénomènes sociaux, politiques, économiques et sociologiques, plutôt que les traits intemporels, tels que le paysage ou le climat. Il ne révèle rien du destin individuel de l'auteur, qui n'apparait dans le reportage que comme celui qui, par divers moyens, cherche à rendre visible l'objet de son étude.

Dans la littérature suédoise, les "écrivains-reporters ", c'est-à-dire les romanciers, dramaturges ou poètes reconnus qui ont écrit des reportages, sont étonnamment nombreux. Le genre du « reportage d'écrivain » a été pratiqué en Suède pendant tout le $\mathrm{xx}^{\mathrm{e}}$ siècle. Ce genre repose, évidemment, sur le postulat selon lequel on peut, d'une façon ou d'une autre, au moins partiellement, montrer la réalité au moyen du langage, faute de quoi on peut être écrivain, mais non reporter. Toutefois, dans la pratique, les écrivains-reporters se trouvent fréquemment confrontés à une série de difficultés inhérentes à l'écriture réaliste, par exemple à l'opposition entre réalité et apparence, ou à la question de l'interprétation des faits observés. Il est rare qu'ils se livrent à de longues réflexions théoriques sur les difficultés de leur entreprise, mais l'existence de ces difficultés transparaît souvent à travers leurs textes. 
3 Jörn Donner est l'un des écrivains-reporters les plus importants de la seconde moitié du $\mathrm{xx}^{\mathrm{e}}$ siècle. Je me propose de présenter ici son Rapport från Donau («Rapport du Danube »), qui n'est pas le plus célèbre de ses reportages, mais qui met nettement en évidence les obstacles auxquels se heurte celui qui aspire à faire apparaître le réel.

4 Jörn Donner est Finlandais, et appartient à la minorité suédophone de Finlande. Il est né en 1933. Il a fait ses débuts en littérature dès l'âge de 18 ans, en 1951. Il a aussi été cinéaste et critique de cinéma (la seule de ses œuvres qui ait jamais été traduite en français est son étude sur les films d'Ingmar Bergman ${ }^{1}$ ). Il a également été homme politique, conseiller municipal communiste, député libéral et, après l'adhésion de son pays à l'Union Européenne, il a siégé au Parlement de Strasbourg.

5 Au centre de son œuvre, qui présente donc une grande richesse et une grande variété de formes, il y a quelques grands thèmes, en eux-mêmes peu originaux, mais dont il montre concrètement le rôle déterminant dans le destin des individus singuliers et de l'être humain en général, et dont les plus importants me paraissent être la liberté, l'attachement au pays natal et l'Europe.

6 Ces thèmes sont présents dans son premier roman marquant, paru en 1955, Jag, Erik Anders $^{2}$ («Moi, Erik Anders»), un genre de Bildungsroman, de roman de formation en raccourci, à bien des égards autobiographique. On y voit un jeune Finlandais d'une vingtaine d'années qui s'efforce de sortir du milieu bourgeois et conformiste dans lequel il a grandi. Pour tenter de forger sa propre identité, de connaître le monde et d'y trouver sa place. Il voyage pendant quelques mois à travers l'Europe. Il quitte Helsinki au printemps 1953. Il séjourne en Italie, puis il se rend à Vienne et Budapest, où il apprend, en lisant des journaux occidentaux, la révolte des ouvriers de Berlin-Est qui commence le 17 juin. En même temps que l'évolution d'Erik, le roman présente celle de l'Europe, dont l'avenir semble alors tout aussi ouvert que celui du personnage principal, qui a l'essentiel de son destin devant lui.

7 Erik veut prouver "qu'on peut être en même temps européen et profondément national ${ }^{3}$ ». En cela, il est sans aucun doute le porte-parole de l'auteur. La quête d'une identité individuelle et l'observation de l'Europe sont deux entreprises qui ne peuvent être menées séparément : tel est le postulat à partir duquel s'élaborent les livres de reportages qui ont permis à Jörn Donner de se faire connaître du grand public, dont le premier, écrit en 1958, est Rapport från Berlin ("Rapport de Berlin »), qui présente le Berlin de 1957-58, une ville déjà divisée en secteur occidental et secteur oriental, mais où le mur n'a pas encore été construit et où l'on passe relativement facilement d'un secteur à l'autre. Ce livre a aujourd'hui encore, outre sa valeur littéraire, une valeur documentaire. Il se montre critique envers le camps occidental, mais ne prend pas parti pour l'Est ${ }^{5}$.

8 À la suite du succès du «Rapport de Berlin », et dans le même esprit, il publie en 1962 Rapport från Donau ${ }^{6}$ («Rapport du Danube »), écrit entre octobre 1958 et août 1962, qui fait la synthèse de lectures, de recherches, d'interviews et de plusieurs voyages et séjours en Europe Centrale, à Vienne, Prague et Budapest, mais aussi à Sarajevo.

9 Dans une courte préface, Donner expose l'objet de ce « rapport». Il s'agit de montrer l'Europe Centrale telle qu'elle est en 1962, et d'essayer d'expliquer par l'histoire la situation présente. Pour tout Européen, « apprendre quelque chose sur ce monde, c'est apprendre quelque chose sur son propre passé.» (Donau, p. 5) Le livre s'inscrit dans un projet plus large d'étude du continent et de sa civilisation, étude qui doit lui permettre 
de mieux comprendre et connaître sa patrie. L'enquête sur l'Autriche, la Tchécoslovaquie et la Hongrie est étroitement liée à sa propre identité : "J'étais étranger parce que les pays dont je traite surprennent par leur différence, par des comportements et des normes inusités. J'étais dans un milieu familier parce que je suis Européen. Je suis porteur du même héritage et j'ai en partie les mêmes mœurs. » (Donau, p. 5)

10 Donner déclare que son «Rapport du Danube » est subjectif : «Le récit constitue un choix subjectif de réalités vécues, réalités du voyage et réalités des lectures. " (Donau, p. 5) Il n'en dit pas plus sur la question de l'objectivité et de la subjectivité. Le caractère subjectif du texte peut lui-même tenir à des raisons subjectives, un parti pris de l'auteur, ou objectives, l'impossibilité, quel que soit le moyen d'expression retenu, de montrer les choses d'une manière parfaitement objective.

11 L'objet étudié par Donner, les pays danubiens en 1962, semble particulièrement bien se prêter à une démonstration par l'exemple des difficultés qu'il y a à rendre compte du réel. La coexistence, sur le sol de l'Europe, de deux systèmes politiques radicalement différents fait qu'un seul et même phénomène donne souvent lieu à deux interprétations, deux descriptions radicalement différentes. Des écrans, de nature diverses, s'interposent entre le regard et la chose regardée. L'écrivain-reporter est à la fois l'observateur qui perçoit l'image transmise à travers un écran et le chercheur, l'investigateur qui a conscience ou prend conscience de la présence de cet écran, qu'il tente d'éliminer.

12 Il serait toutefois illusoire de croire qu'un voyageur n'ayant jamais entendu parler du lieu qu'il va visiter serait plus apte qu'un autre à en avoir une vision correcte. Jörn Donner rapporte qu'en 1953 il s'est promené au Prater, à Vienne. Dans cet immense parc, il n'y avait alors quasiment rien à voir, les stands étaient abandonnés, les allées désertes, le vent faisait voltiger des morceaux de vieux journaux. «Et le présent, c'est un grand parc d'attractions qui paraît mort en l'absence d'êtres humains. Le quartier du Prater fait partie du secteur russe. » (Donau, p. 33) Pour appréhender véritablement la réalité du Prater, il faut connaître son histoire, la place qu'il a tenu dans la vie viennoise, il faut savoir ce qui s'y est autrefois passé, il faut aussi avoir lu des romans qui l'évoquent ou, par exemple, vu le film d'Orson Wells Le troisième homme. (Cf. Donau, p. 32-33.)

13 Mais un touriste qui se rend pour la première fois à Vienne vers 1960 n'y arrive évidemment pas l'esprit vierge de toute représentation a priori. Les visites guidées, au lieu de montrer la ville telle qu'elle est, ne font que confirmer les idées préconçues, selon lesquelles on y vit toujours comme en 1914, et l'existence, réglée par une hiérarchie sociale et des convenances strictes, se déroule pour l'essentiel entre les salons de la Hofburg, le château de Schönbrunn et l'Opéra. Le tourisme étant une des ressources essentielles de l'économie autrichienne, il importe avant tout de répondre aux attentes de la clientèle: «On ne se préoccupe pas du fait que la partie de la réalité que montrent les monuments n'est qu'une toute petite facette de l'histoire.» (Donau, p. 37) Dans le tourisme, deux écrans qui rendent impossible une perception objective du réel conjuguent leurs effets, ou même, coïncident : le jugement que porte a priori l'observateur, et la mise en scène d'elle-même à laquelle procède la chose observée.

14 Les grandes œuvres littéraires, quant à elles, sont parfois, pour d'autres raisons, aussi trompeuses que les brochures publiées par l'office du tourisme de la république autrichienne. Ni Joseph Roth, ni Stefan Zweig n'ont compris l'évolution de leur pays 
après 1918. Lorsque Roth, dans Die Kapuzinergruft (écrit en 1938), voit dans le principe monarchique et le rassemblement de tous les patriotes le salut de l'Autriche, il fait preuve d'une naïveté qui sera cruellement corrigée par l'histoire un an avant sa mort, qui survient en mai 1939. Chez Zweig aussi, on retrouve l'idée selon laquelle, après la Première Guerre mondiale, la monarchie, telle qu'elle avait existé jusqu'en 1918, aurait pu sauver l'Autriche du nazisme. "Aux ennemis présents de l'esprit, il n'avait rien d'autre à opposer qu'un idéal mort depuis longtemps. » (Donau, p. 77) Stefan Zweig, comme Joseph Roth, donne une image erronée de l'Autriche entre les deux guerres mondiales, parce qu'il n'a pas compris ou n'a pas voulu comprendre que le passé était une époque révolue et que son univers mental est resté celui de ce passé, alors que les temps avaient profondément changé (cf. Donau, p. 77).

La substitution du passé au présent reste selon Donner un trait caractéristique de la Vienne du début des années 1960. Il écrit à propos des gardiens de la Hofburg: "des fonctionnaires sans âge. Parfois, je crois qu'on les sort des armoires et qu'on les nettoie chaque année en début de saison. » (Donau, p. 22) Partout, du bal de l'opéra aux étalages de cartes postales, dont un grand nombre représentent l'empereur François-Joseph, le passé vient se superposer au présent et le rendre invisible. Il sert de refuge, car il est généralement vu comme « le bon vieux temps » (Donau, p. 17).

Mais ce qualificatif de «bon vieux temps » est mensonger. Une grande partie du prétendu passé qui s'exhibe à Vienne n'a en fait jamais existé. La Première République et la période nazie ont été tout sauf des époques heureuses, et, sous la Double Monarchie, seul un petit groupe d'individus avait la possibilité de mener une vie agréable et insouciante (cf. Donau, p.17). "Cette vision d'une réalité passée, qui se reflète dans les valses, les opérettes, les chansons, n'est pas l'expression d'un monde réel. C'est un monde de rêve. » (Donau, p. 16) Ce qui empêche de voir la réalité présente est moins le passé proprement dit que la représentation déformée qui en est donnée, et qui trompe d'autant mieux qu'elle est composée d'éléments véridiques.

Vers 1960, le bal de l'Opéra se déroule toujours selon un rituel traditionnel tellement précis que l'événement semble comme irréel, car il y manque la dimension de l'inattendu, qui distingue habituellement le vécu des constructions mentales. Ainsi, un journaliste d'un grand quotidien peut écrire l'article qui raconte le bal avant que celuici n'ait eu lieu (cf. Donau, p. 23). L'image, ici, fait plus que dissimuler la réalité, elle la modèle et la remplace.

18 La tromperie, par la parole, par l'apparence, par la mise en scène, se pratique dans de multiples domaines, futiles ou sérieux. Le Danube est qualifié de bleu, bien qu'il ne le soit jamais. Dans les immeubles d'habitation, l'étage appelé "premier étage » est le quatrième niveau au-dessus du rez-de-chaussée (cf. Donau, p. 40). «Plus la vieille noblesse perdait son véritable pouvoir, plus elle accordait d'importance à la pompe et à la décoration.» (Donau, p. 25) « Partout à Vienne on trouve ce manque de congruence entre la réalité et les rêves, entre la vie et la façade.» (Donau, p. 41) Donner cite l'architecte autrichien Adolf Loos, qui, en 1898, qualifiait Vienne de "Potemkinstadt ", "ville à la Potemkine »" (cf. Donau, p. 43), et un biographe de Loos, qui déclare que "L'homme [...] de l'époque de François-Joseph éprouvait, dans son sentiment d'insécurité intérieure, une peur mortelle de la réalité. » (Donau, p. 44) Le colonel Alfred Redl, homme raffiné et brillant, irréprochable officier d'état-major de l'armée impériale et royale, était en réalité un espion à la solde de la Russie (cf. Donau, p. 66-72). L'Autriche ayant été placée en 1945 par les Alliés dans la catégorie des pays qui avaient 
été occupés par l'Allemagne nazie, "elle a été soudainement libérée du sentiment d'être co-responsable des crimes du nazisme. » (Donau, p. 147)

Jörn Donner, pour sa part, voit en Hitler un Autrichien, un homme qui a été marqué par Vienne. Il y a quelque chose de viennois dans le nazisme, dans la mesure où il dissimule la réalité par des mots qui suggèrent autre chose que ce qu'ils désignent en fait : «le nazisme [...] appelle l'assassinat des juifs die Endlösung [«la solution finale»], la politique de la terreur la pacification, et les conquêtes extension du Lebensraum [" de l'espace vital »]. Ces euphémismes visent à donner une impression d'humanité. Des accents mélodramatiques s'alliaient à la cruauté. » (Donau, p. 150)

Le « Rapport du Danube » de Jörn Donner met en évidence, les uns après les autres, les masques qui recouvrent le véritable visage de l'Autriche. Il informe ainsi sur ce que l'Autriche n'est pas. Mais informer sur ce qu'elle est paraît être une tâche plus difficile, comme si l'abondance et l'épaisseur des masques avaient fini par effacer presque totalement le visage lui-même. Donner semble voir un portrait assez fidèle de l'Autrichien contemporain dans le personnage de Herr Karl, créé à la télévision par l'acteur et artiste de cabaret Helmut Qualtinger, héritier spirituel de Nestroy et Raimund. Herr Karl est un être passif et opportuniste, qui cherche d'abord à vivre sans soucis. Après 1918, il a été successivement socialiste, austro-fasciste et nazi, et il a ensuite acclamé les Russes, puis les Américains. «Herr Karl est une autocritique pertinente et terrible de l'homme sans qualités, le contraire du héros intellectuel de Musil, qui savait malgré tout choisir. Herr Karl ne choisit pas. Il se renie lui-même, y compris ce qu'il y a de bien en lui-même. » (Donau, p. 51) « La mollesse de Herr Karl, sa Schlamperei, son amabilité (qui ne l'empêche pas d'être cruel), c'est l'Autriche vue dans un miroir sombre, l'Autriche transformée en une incarnation de l'impuissance.» (Donau, p. 53) En résumé, Herr Karl est « un homme qui n'est rien » (Donau, p. 54).

21 Lorsqu'il entreprend de caractériser celui qui serait l'Autrichien typique, Donner peut parfois sembler utiliser davantage le personnage créé par Helmut Qualtinger que ses propres constatations. Dans les pages consacrées à Vienne, on ne retrouve pas vraiment l'abondance d'observations et de détails concrets qui était l'un des traits marquants du «Rapport de Berlin». Dans le Berlin d'après 1945, le passé, considéré comme totalement négatif, était toujours tenu à distance, par le reporter comme par ses interlocuteurs. Le présent marquait le début d'une époque totalement autre, dont il n'existait quasiment encore aucune image dans la littérature ou l'histoire, et qu'il importait donc d'examiner avec minutie. De Vienne, il y a au contraire une profusion d'images, dont sont imprégnés à la fois les Viennois eux-mêmes et le Finlandais venu les regarder, et qui risque sans cesse de substituer ses représentations a priori aux faits.

Donner comprend que le portrait de l'Autrichien en Herr Karl doit bien entendu être nuancé, faute de quoi il ne serait lui aussi qu'un stéréotype supplémentaire. Il y a des Autrichiens qui ne ressemblent pas à Herr Karl, et « la critique exprimée par la pièce ne se limite pas à la patrie de Herr Karl. » (Donau, p. 51) En outre, Qualtinger, le créateur de Herr Karl, est lui-même Viennois, et «patriote local ». Sa ponctualité, son sérieux, son sens de l'objectivité n'ont, selon Donner, rien d'autrichien, mais «il aime la bonne chère et le bon vin. Son ironie envers lui-même se mêle d'apitoiement sur lui-même, son agressivité d'amabilité. » (Donau, p. 54) Par une sorte de retournement dialectique, il devient « un exemple de la manière dont le caractère viennois peut se revivifier en se reniant lui-même. »(Donau, p. 54) 
Donner condamne la Vienne qui n'est qu'un décor, mais la place importante qu'occupe dans le livre l'ancienne capitale des Habsbourg parait indiquer qu'il n'échappe sans doute pas complètement au charme qu'elle exerce. Cette attirance surprenante peut s'expliquer par l'intérêt, exprimé dans le roman autobiographique Jag, Erik Anders, pour des milieux de vie différents de celui dans lequel il a grandi, et plus généralement par le désir de trouver, paradoxalement, derrière ce qui a l'apparence du faux et de l'absurde, une vérité plus profonde, que ne percevrait pas un regard étroitement rationaliste. Mais comme le sens critique de Donner reste en même temps trop fort pour qu'il puisse durablement être abusé, son « Rapport du Danube " s'élabore en partie dans une tension entre fascination et colère et sera, comme il le déclare en 1976, de tous ses livres, celui qui a été le plus difficile à écrire ${ }^{8}$.

nas surprenant que Donner, qui s'attache à dénoncer les trompe-l'œil qui recouvrent la réalité autrichienne, éprouve de l'admiration envers les Autrichiens qui, dans le présent ou le passé, se sont fixé ce même objectif : Adolf Loos, qui entreprit de remplacer les façades surchargées et pompeuses par une architecture fonctionnelle, toujours méconnu dans son propre pays au moment où Donner écrit, dont certaines constructions ont été rasées, Sigmund Freud, qui explorait implacablement la part cachée de l'âme, et qui portait sur l'être humain et la société un regard pessimiste, mais dont L'interprétation des rêves peut être lue " comme une étude sociologique de la Vienne du passé. » (Donau, p.130) Donner estime qu'en 1962 «Freud inspire toujours la même aversion aux propagateurs du mensonge vital de Vienne que celle qu'il inspirait au nazisme. Sa conscience lucide, sa volonté de savoir sont à l'opposé des décors et des mises en scène mystificatrices de la bourgeoisie viennoise. » (Donau, p. 131)

Enfin, parmi ceux que Donner admire, il y a Egon Erwin Kisch, le journaliste de génie qui révéla au public la trahison du colonel Redl, un analyste dépourvu de préjugés et de dogmatisme. "Par sa culture aux multiples facettes, sa curiosité sans relâche, son regard ouvert sur le monde entier, Kisch était un produit de ce qu'il y avait de meilleur dans l'Autriche-Hongrie, d'une tradition que le temps et l'histoire ont contribué à effacer. » (Donau, p. 74)

Les images mensongères de la réalité ne sont pas inamovibles, elles peuvent être brisées. Elles produisent même dans certains cas l'effet inverse de celui qui était recherché : plus elles sont nombreuses et fortes, plus elles suscitent de vocations d'iconoclastes. Ainsi, Vienne s'avère être aussi un lieu qui a fait naître des regards radicalement nouveaux sur le monde.

Dans les pays à régime communiste, les écrans destinés à déformer ou dissimuler des faits sont souvent faciles à déceler. L'un des premiers obstacles à la perception de la réalité est précisément la croyance, sincère, naïve, ou cyniquement feinte, en l'existence d'un discours qui serait la vérité objective. Ainsi, en Tchécoslovaquie, depuis 1948, «Seule une interprétation de l'histoire est acceptée. Elle ne s'appelle pas interprétation de l'histoire, mais vérité sur le passé. » (Donau, p. 161)

28 Là où un régime communiste est en place, il existe aussi une image du présent qui semble être devenue tout à fait indépendante de la réalité présente, et constituer un monde imaginaire, cohérent mais auto-référentiel: «Depuis quatorze ans, les dirigeants tchèques font l'éloge des remarquables dirigeants tchèques. » (Donau, p. 160) La vie dans une démocratie populaire, estime Donner, exige des talents d'acteurs. Le comportement des citoyens dans l'espace public ne fournit à l'observateur extérieur 
aucune indication sur leurs opinions ou leurs sentiments. C'est seulement en privé qu'ils enlèvent leur masque. Tous semblent alors plus ou moins opposés au régime, et le reporter constate : « plus je rencontre de gens en privé, plus je me demande où sont les véritables partisans du régime. » (Donau, p. 198)

On pourrait donc avoir l'impression qu'il existe en Tchécoslovaquie et en Hongrie une opposition politique, seulement empêchée de se manifester ouvertement par la répression. Mais cette image-là est elle aussi trompeuse. Si la critique du régime en place est très répandue, personne ne paraît, en 1962, réfléchir à ce par quoi il serait souhaitable de le remplacer. Ceux qui ont perdu leur statut social idéalisent le passé, plus que des opposants, ce sont des rêveurs : "Sans pouvoir, sans influence, une partie de ces gens rêvent d'une restauration du passé, mais ne peuvent rien faire pour leurs idées, à part écouter des stations de radio occidentales. » (Donau, p. 189)

À ces rêveurs s'oppose la figure du dernier grand homme d'Europe Centrale évoqué par le livre, le philosophe Georg Lukács, auquel Donner a rendu visite à Budapest. Il est désormais isolé, car il a cessé d'être membre du parti communiste, a fait partie du gouvernement Nagy en $1956^{\circ}$, et est considéré comme un "révisionniste » par les représentants du système communiste, mais n'est pas suffisamment opposé au communisme et n'a pas été suffisamment persécuté par le régime pour pouvoir bénéficier de la sympathie des dirigeants occidentaux. Pour Donner, il est « l'humaniste solitaire » (Donau, p. 240), et «Il n'y a qu'un seul endroit à Budapest où l'on trouve un humanisme universel. C'est le bureau de Lukács.» (Donau, p. 227) Ce génie solitaire rappelle par certains traits ceux qui ont été évoqués dans les pages consacrées à Vienne.

31 Cette ressemblance n'est sans doute pas fortuite puisque l'on voit peu à peu, au fil des pages, se dessiner une troisième image de la Hongrie et de la Tchécoslovaquie, déterminée cette fois par le désir du reporter lui-même, qui souhaite pouvoir constater que le passé commun aux pays qui constituaient jadis ensemble l'Empire des Habsbourg doit, en 1962 encore, se manifester par des similitudes importantes entre ces pays, malgré les différences de système politique. Après la préface à son «Rapport du Danube », Donner a d'ailleurs placé une carte politique du centre de l'Europe sur laquelle figurent à la fois les frontières de 1962 et, en gris sur fond blanc, celles de l'Empire austro-hongrois en 1914. Il entend montrer la persistance, au niveau de la culture, des traditions, des mentalités, d'une unité danubienne. En prenant son petit déjeuner dans un café de la place Venceslas, à Prague, en tous points semblable aux cafés de Vienne, il peut s'identifier au narrateur d'un roman de Joseph Roth qui, lorsqu'il pénètre en 1914 dans l'unique café de Zlotograd, aux confins de l'Empire, a l'impression de se retrouver dans le café qu'il a l'habitude de fréquenter dans la Josefstadt, à Vienne (cf. Donau, p. 260-261). Cette volonté de mettre en avant le passé commun explique la part importante qu'occupent les rappels historiques dans le livre. Ceux-ci sont nombreux, même dans la dernière partie, intitulée "Voyage d'aujourd'hui ».

32 Jörn Donner explique l'accession de la Hongrie et de la Tchécoslovaquie à l'indépendance après 1918 par la montée de nationalismes de plus en plus forts à l'intérieur de l'Empire, nationalismes qui, selon lui, dans les petits pays, sont légitimes, car ils y sont toujours associés à d'autres exigences, comme celles de meilleures conditions de vie ou de plus de justice sociale. Contrairement à ce qu'affirmaient parfois des représentants des vieux États-Nations, le nationalisme des petits pays n'est 
pas condamnable. On voit ici bien sûr Donner porter un regard de Finlandais sur l'Europe Centrale, dont les mouvements de libération nationale "eurent aussi pour conséquence indirecte l'indépendance de la Finlande " (Donau, p. 82). L'apparition de nouveaux Etats souverains sur le territoire de l'ancienne double monarchie est ainsi comprise comme l'aboutissement de l'évolution interne de cette monarchie.

La présentation historique de l'Europe Centrale conduit toutefois inévitablement Donner à évoquer les causes du passage au communisme de tous les pays danubiens, à l'exception de l'Autriche. Il est alors contraint de constater qu'elles n'ont rien à voir avec l'histoire de l'Autriche-Hongrie. Les systèmes politiques en place dans les pays dits «de l'Est » sont une conséquence directe du partage de l'Europe après 1945, partage face auquel les petits pays sont apparemment sans pouvoir. La réalité que Donner a sous les yeux en 1962 est l'effet direct du rattachement de la Hongrie et de la Tchécoslovaquie au bloc communiste, qui marque profondément les sociétés qui y appartiennent. À l'issue de son investigation, Jörn Donner doit donc renoncer à son hypothèse de départ, et admettre que l'Empire décrit par Joseph Roth a totalement disparu. La troisième image de la Tchécoslovaquie et de la Hongrie, comme pays qui seraient toujours culturellement semblables à l'Autriche, est réfutée à son tour. À la fin $\mathrm{du}$ travail de reportage, il ne reste rien ni de l'image flatteuse du régime communiste que tentent d'en donner ses dirigeants, ni de l'image qu'en ont ses victimes, ni de l'image qu'en avait l'auteur lui-même. Toutes ces images étaient illusoires.

En 1962, la Tchécoslovaquie et la Hongrie sont d'abord et avant tout des dictatures, dans lesquelles le pouvoir en place détermine même la vie quotidienne des citoyens. Ceux-ci, quelles que soient leurs velléités ou leur volonté, n'ont pas la possibilité de transformer profondément le régime sous lequel ils vivent, qui est le produit, non de données nationales, mais de la situation politique internationale. «La tension dans le monde a un effet direct sur le niveau de vie à l'Est, car l'armement augmente en même temps que la tension. L'exacerbation de la guerre froide signifie que la liberté du particulier est encore plus réduite, que moins d'Européens de l'Ouest ont l'occasion de se rendre en visite à l'Est, que l'Europe de l'Est communiste s'isole. » (Donau, p. 245-246) Le livre se termine par une prise de conscience de l'auteur, à la fois bilan et conséquence du reportage : l'ancien monde danubien n'existe plus. "Quatorze années de régime communiste en Hongrie et en Tchécoslovaquie ont balayé la plupart des traces du passé commun. » (Donau, p. 261)

Ce bilan a une dimension tragique pour Donner, car il a laissé entendre dans sa préface, et on comprend au fil des pages que l'Europe Centrale représente symboliquement la totalité de l'Europe, dont il avait à cœur de montrer que, malgré ses multiples divisions, elle formait une entité culturelle originale. Dans le chapitre 14 de la troisième partie (Donau, p. 199-201), il n'est pas question des pays danubiens, mais de l'Europe et de sa division. À plusieurs reprises, dans le reste du livre, on voit, à l'intérieur d'une même phrase ou d'un même paragraphe, d'un même raisonnement, la notion d'Europe se substituer à celle d'Europe Centrale. Donner déclare ainsi dans sa conclusion: "Je voulais écrire un livre sur Vienne, Prague et Budapest, sur l'Europe Centrale. Je n'avais qu'une seule thèse. Contre ceux qui proclamaient la division définitive de l'Europe, je voulais proclamer l'unité de l'Europe.» (Donau, p. 260, les italiques sont de moi.) Il avait écrit auparavant à propos de Ken, un Américain : « Il considère Vienne avec amusement, avec une légère indulgence. Il sait et sent que son État est plus efficace, plus riche, plus vigoureux et plus dur que l'Europe.» (Donau, p. 30, les italiques sont de moi.) 
peut évidemment remarquer que l'Europe Centrale joue dans le «Rapport du Danube » le rôle d'un écran à travers lequel Jörn Donner voit l'Europe. La substitution de l'une à l'autre est ici toutefois légitime, dans la mesure où elle est consciente et délibérée et où l'auteur l'a annoncée et justifiée dès sa préface.

Comme les hommes d'Europe Centrale qu'il admire, Sigmund Freud, Georg Lukács, Egon Erwin Kisch, un modèle en matière de journalisme, Jörn Donner a un parti pris de lucidité, et sait admettre des vérités qui lui déplaisent. La division, et la décadence de l'Europe sont pour lui des faits qui ne peuvent être niés. Mais reconnaître les choses telles qu'elles sont ne signifie pas s'y soumettre avec résignation. Chez Donner, il n'est pas rare qu'il y ait une protestation de l'âme, peut-être inopérante, mais clairement formulée, contre ce qui s'est révélé au regard iconoclaste. Ainsi, lorsqu'il a expliqué quel jugement l'Américain Ken porte sur l'Europe, il poursuit :

Le pire est qu'il a raison dans de qu'il dit. L'Europe est petite, éclatée en petits États [...] Beaucoup d'Européens continuent à s'imaginer qu'ils vivent au centre du monde. Ils croient qu'en dehors de la civilisation européenne il n'y a pas de salut, que la culture européenne est la culture universelle.

Et pourtant je réagis contre Ken. Il mesure l'importance d'un phénomène à sa taille. Si sa civilisation est la réponse à la civilisation européenne, alors je préfère mon propre continent, avec sa vanité. (Donau, p. 30)

Donner sait que l'Europe se trouve désormais à la périphérie du monde. Comme les pays qui occupent son centre, elle pourrait bien n'être plus qu'une superposition de toiles peintes qui recouvrent surtout du vide, un monde qui n'est plus, d'où émergent quelques figures de génies solitaires. Mais c'est en Europe, selon lui, et tout particulièrement dans la région danubienne, qu'ont pris naissance les phénomènes qu'on voit, au début des années 1960, se développer sur les autres continents, comme par exemple les mouvements de libération nationale. Il laisse ici implicitement entendre que l'Europe a joué un rôle particulier dans l'évolution de l'humanité, pour le meilleur ou pour le pire ${ }^{10}$.

C'est avec un même parti pris de lucidité qu'il conclut son livre, en constatant le caractère irrémédiable de la division de l'Europe, contre laquelle les populations ne peuvent rien, mais en exhortant aussi les Européens à ne pas s'accommoder de cet état de fait. Tout en prenant clairement parti pour ou contre certaines pratiques ou attitudes, il avait refusé de combattre dans l'un des deux camps de la guerre froide, au risque de se retrouver isolé. "Il est difficile ", écrivait-il, " de faire partager son point de vue si, comme moi, on approuve en principe certaines conceptions du régime [communiste] en matière de grandes orientations sociales, mais que l'on s'oppose en même temps au fait que la sécurité et la liberté d'opinion de l'individu ne soient pas assurées.» (Donau, p. 199) Il demande pour finir aux Européens de l'Ouest de ne pas oublier l'existence de « l'autre Europe »:

Notre Europe est traversée par une frontière, par des barbelés, un mur et des tranchées, des champs de mines.

Voilà le fait tout simple qui brise les illusions que je me faisais sur son unité.

La position que j'occupe entre ces mondes, je l'ai peut-être indiquée dans ce livre, mais cela ne signifie pas que je veuille cesser de regarder par-dessus la frontière. Le paradoxe est en effet que, malgré l'hostilité et la méfiance, nous devons sérieusement apprendre à vivre avec cette autre Europe. Ne pas le faire reviendrait à abandonner tout espoir. (Donau, p. 261-262)

"Mais raconter l'histoire de ces villes ", écrivait Donner en introduction, "c'est tout autant raconter ma propre histoire, depuis le jour où je suis arrivé pour la première fois 
à Vienne, Budapest et Prague, jusqu'au jour où j'écris ceci. » (Donau, p. 5) Il pourrait sembler que le texte contredit cette affirmation, puisqu'il ne contient aucune allusion à la vie personnelle de l'auteur. En fait, il n'est pas question ici de retracer un destin individuel, mais de découvrir une identité culturelle. Le "Rapport du Danube » va cependant au-delà de l'intention première exposée dans la préface. Il raconte la découverte d'une disparition, d'une absence, et le cheminement intérieur de celui qui voit ses convictions réfutées par les faits. Ce qui est remarquable, dans le reportage de Donner, c'est entre autre que l'évolution intellectuelle qu'il retrace n'est pas relatée après coup par un narrateur qui connaîtrait lui-même déjà la fin de l'histoire lorsqu'il en écrit le début, mais qu'elle se fait dans une large mesure en même temps que l'écriture, qui suit elle-même de très près le travail de recherche. Le reportage est en relation plus immédiate avec le réel que la plupart des œuvres narratives, qu'il s'agisse de fictions ou d'autobiographies, ou même de récits de voyages, qui peuvent être écrits plusieurs années après le retour du voyageur dans son pays. En se faisant reporter, l'écrivain accepte de confronter directement sa vision du monde au monde.

\section{NOTES}

1. Donner, Jörn: Djävulens ansikte. Ingmar Bergmans filmer, Stockholm: Aldus/Bonnier, 1962. Traduction française : Ingmar Bergman, traduit par Frostenson, Sven, adapté par Braucourt, Guy, Paris : Seghers, 1970. Nouvelle édition remaniée en 1973.

2. Donner, Jörn : Jag, Erik Anders, Stockholm : Wahlström \& Widstrand, 1955.

3. Ibid. p. 149.

4. Donner, Jörn : Rapport från Berlin, Helsingfors : Söderström \& Co, 1958, 240 p. Nouvelle édition en 1966, avec une préface nouvelle, Stockholm : Wahlström \& Widstrand.

5. « Rapport de Berlin » n'était acceptable pour aucune des deux Allemagnes. Cela peut expliquer pourquoi ce livre n'a jamais été traduit en allemand, estime l'auteur en 1986, sans doute à juste titre. Cf. Donner, J. : Jörn Donner Tyskland, Helsingfors, 1998, p. 23.

6. Donner, J. : Rapport från Donau, Stockholm : Bonnier, 1962, 271 p. Dans ce qui suit, je désigne ce livre par l'abréviation Donau.

7. Cette expression fait allusion aux "villages de Potemkine», villages russes situés sur le parcours d'un voyage que devait effectuer la tsarine Catherine II, et dont Potemkine avait pris soin de faire décorer les façades afin de dissimuler leur misère.

8. Cf. Donner, J. : Sagt och gjort, Helsingfors : Söderström \& Co, 1976, p. 54.

9. Le gouvernement d'Imre Nagy avait été mis en place en Hongrie à la suite de la révolte d'octobre 1956 contre le régime de type soviétique, et avait tenté de modifier profondément ce régime. Il prit fin en novembre de la même année, à la suite de l'intervention militaire de l'URSS. 10. Cette idée, qui apparaît ici implicitement, sera plus tard développée par Donner dans un autre livre de reportage, Världsboken. Ett reportage («Le livre du monde. Un reportage»), paru à Stockholm chez Wahlström \& Widstrand en 1968. Je renvoie à ce propos à mon article « Regards d'écrivains-reporters suédois sur l'Asie dans les années 1960 », in Auchet, Marc: Les pays nordiques et le dialogue interculturel, Nancy : Presses Universitaires de Nancy, 1999, p. 219-240. 


\section{RÉSUMÉS}

Le Finlandais Jörn Donner est écrivain, cinéaste et critique de cinéma. Il a écrit des romans, des nouvelles et des essais, mais aussi une série de livres de reportage, qui font de lui l'un des «écrivains-reporters » de langue suédoise les plus marquants de la seconde moitié $d u x^{e}$ siècle. Son « Rapport du Danube », paru en 1962, est une tentative pour briser les images toutes faites de l'Autriche, de la Hongrie et de la Tchécoslovaquie et pour montrer la persistance, malgré la division politique de l'Europe, d'une culture commune aux pays d'Europe centrale. L'entité culturelle constituée par l'Europe centrale représente, par métonymie, l'ensemble de l'Europe. Mais l'enquête sur le terrain fait peu à peu apparaître que la division des territoires de l'ancienne monarchie danubienne est plus profonde que ne l'avait supposé le reporter avant d'entreprendre son reportage.

Der Finländer Jörn Donner ist Schriftsteller, Filmemacher und Filmkritiker. Er hat Romane, Erzählungen und Essays geschrieben, aber auch eine Reihe von Reportagebüchern, die ihn zu einem der bedeutendsten schwedischsprachigen « Schriftstellerreporter » der zweiten Hälfte des 20. Jahrhunderts machen. Sein 1962 erschienener Rapport från Donau («Bericht über die Donauländer») ist ein Versuch, die gängigen Bilder Österreichs, Ungarns und der Tschechoslowakei zu durchbrechen und der Teilung Europas zum Trotz auf das Weiterbestehen einer gemeinsamen mitteleuropäischen Kultur hinzuweisen, wobei die kulturelle Einheit Mitteleuropas metonymisch ganz Europa vertritt. Im Laufe der Untersuchung erweist sich jedoch die Teilung der Donauländer als tiefgreifender als der Reporter es eingangs vorausgesetzt hat.

\section{AUTEUR}

\section{ANNIE BOURGUIGNON}

Université Nancy 2 